\title{
Accelerometer assisted Electromagnetic Positioning System Design
}

\author{
Zhengping $\mathrm{Li}^{1, \text { a }}$, Long Zhao ${ }^{1, \mathrm{~b}}$ \\ ${ }^{1}$ Department of Electrical and Information Engineering, North China University of \\ Technology,Beijing, 100044, China \\ aemail:lizp@ncut.edu.cn, bemail:764796185@qq.com
}

Keywords: Positioning; Electromagnetic System; 3-axis Accelerometer

\begin{abstract}
In the electromagnetic positioning system, it was found that with the increase of distance between the target carrier and the emission source, positioning accuracy decreased a lot. This paper proposed a method that used 3-axis accelerometer to assist the electromagnetic positioning system to measure the position of the target. The simulation results showed that the method could obviously improve the positioning accuracy.
\end{abstract}

\section{Introduction}

Positioning system is a device for spatial position measurement, which is widely used in virtual reality, artificial intelligence, etc [3]. At present, several commonly used methods of positioning are ultrasonic localization, photoelectric positioning, electromagnetic positioning [4]. In this paper, the electromagnetic positioning system employs a magnetic transmitter and a magnetic sensor, both signal one after another. And the 3-axes receiving coils detect three groups of transmitted signals as well as the magnetic field. Then the location of target in space can be computed based on received signal strength and other characteristic values. However, the signal-to-noise ratio of the received signals will be serious decline when the target is far away from the transmitting antenna in practice, which leads to the positioning accurate decrease. The paper introduces a method by using the 3-axis accelerometer to help axes detecting the space position of the target object. The 3-axis accelerometer and the receiving axes are fixed on the target. The linear accelerometer of target can be measured by 3-axis accelerometer and after two integral, the location information can be obtained. According to the space location information from the 3 axes receiving coil and the 3-axis accelerometer, indirect Kalman-Filter is built for data fusion. Then more accurate location information can be obtained.

\section{Design of Coil Measurement System}

Orientation relationship between 3 receiving axes and 3 axes of transmitter is shown in Figure 1,

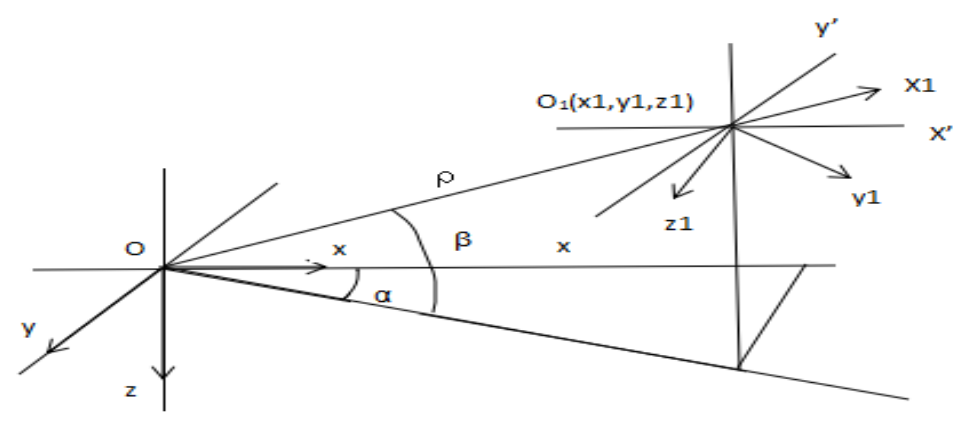

Fig.1. Space coordinate model of emission source and target.

A reference coordinate system $O-x y z$ is established, and the receiver is located at $O_{1}$, In the $O-x y z$

the position coordinate of receiver is $(x, y, z)$, or spherical coordinate $(\rho, \alpha, \beta)$. And the coordinate system $O^{\prime}-x^{\prime} y^{\prime} z$ ' is parallel to the reference coordinate [5][6]. The attitude of receive can be 
described as three successive rotations $w, \psi$ and $\varphi$ with respect to coordinate system $O^{\prime}-x^{\prime} y^{\prime} z^{\prime}$ [2]. The definitions of the three rotations are as follow: first, turning around $z_{1}$ make $w$ degrees of horizontal rotation, then, turning around $y_{1}$ make $\psi$ degrees of elevation rotation, turning around $x_{1}$ make $\varphi$ degrees of roll rotation. The symbols for the rotation angle are specified as follows: observing from the negative half axis of rotation, rotation angle is positive for the clockwise rotation, otherwise, the rotation angle is negative.

The emission source field vector in $O$ is $f_{1}$, and the induced magnetic field in $O_{1}$ is $f_{2}$;

$$
f_{2}=\frac{C}{\rho^{3}} S f_{1}=\frac{C}{\rho^{3}} S T_{\beta} T_{\alpha} f_{0}
$$

And $T_{\alpha}=\left|\begin{array}{ccc}\cos \alpha & \sin \alpha & 0 \\ -\sin \alpha & \cos \alpha & 0 \\ 0 & 0 & 0\end{array}\right|, T_{\beta}=\left|\begin{array}{ccc}\cos \beta & 0 & -\sin \beta \\ 0 & 1 & 0 \\ \sin \beta & 0 & \cos \beta\end{array}\right|$.

In which $S$ is the induced matrix under opposite relation, $S=\operatorname{diag}(1,-0.5,-0.5), C=N I A / 2 \pi, N$ is the turns of transmitter coil, $I$ is the driving current of coil, $A$ is cross-sectional area of transmitter coil. Let $\rho$ be the distance of $O O_{1}$. And the transmit vector matrix $f_{0}=\operatorname{diag}(1,1,1), f_{1}$ : the coordinate system $O$-xyz make $\alpha$ degrees of azimuth rotation and $\beta$ degrees of elevation rotation.

$f_{3}: f_{2}$ make $-\beta$ degrees of elevation and $-\alpha$ degrees of azimuth rotation.

$$
f_{4}=T_{\varphi} T_{\psi} T_{\omega} f_{3}=T_{\varphi} T_{\psi} T_{\omega} T_{-\alpha} T_{-\beta} f_{2}
$$

$f_{4}$ is the vector matrix of receiving antenna. The transverse vectors of $f_{2}$ correspond to the 3 transmit field vectors. And the longitudinal vectors of $f_{2}$ correspond to the 3 magnetic field vectors of receiving source.

$$
P_{2}(z)=\frac{C^{2}}{\rho^{6}}\left|\sin ^{2} \beta+\frac{1}{4} \cos ^{2} \beta\right|
$$

And according to the equivalence relation of $x$ axis and $y$ axis with respect to the $z$ axis,

$$
\begin{aligned}
& {\left[\mathrm{P}_{2}(\mathrm{x})+\mathrm{P}_{2}(\mathrm{y})+\mathrm{P}_{2}(\mathrm{z})\right]=\frac{3}{2} \frac{\mathrm{C}^{2}}{\rho^{2}}\left[\mathrm{x}^{2}+\mathrm{y}^{2}+\mathrm{z}^{2}\right]} \\
& x^{2}+y^{2}+\mathrm{z}^{2}=\rho
\end{aligned}
$$

The result of the left side of the (4) can be obtained by the receiver. So the $\rho$ can be calculated.

$$
f_{4}^{T} f_{4}=\frac{1}{4} \frac{C^{2}}{\rho^{6}}\left|\begin{array}{ccc}
1+3 \cos ^{2} \alpha \cos ^{2} \beta & 2 \sin \alpha \cos \alpha \cos ^{2} \beta & -3 \cos \alpha \sin \beta \cos \beta \\
3 \sin \alpha \cos \alpha \cos ^{2} \beta & 1+3 \sin ^{2} \alpha \cos ^{2} \beta & -3 \sin \alpha \sin \beta \cos \beta \\
-3 \cos \alpha \sin \beta \cos \beta & -3 \sin \alpha \sin \beta \cos \beta & 1+3 \sin ^{2} \beta
\end{array}\right|=\frac{1}{4} \frac{C^{2}}{\rho^{6}} F
$$

So the $\alpha$ and $\beta$ can be calculated:

$$
\begin{aligned}
& \alpha=\arctan \left(F_{23} / F_{13}\right) \\
& \sin ^{2} \beta=\left(F_{33}-1\right) / 3
\end{aligned}
$$

The coordinate of $O_{1}(x, y, z)$ can be expressed as follow:

$$
\begin{aligned}
& x=\rho \cos \beta \cos \alpha \\
& y=\rho \cos \beta \sin \alpha \\
& z=\rho \sin \beta
\end{aligned}
$$

\section{Design of 3-axis Accelerometer Measurement System}

The 3-axis accelerometer can measure the linear acceleration of the target, and motion speed can be obtained through an integral, and the location data can also be gotten through two integral. The 3-axis accelerometer is fixed on the target. So the location information of target can be obtained as well.

The initial position of target is in $O O_{1}$. The distance from $O$ is $s_{1}$.The target begin to move from the rest state along the $O O_{1}$ direction., and the acceleration is $a$. So 


$$
\begin{aligned}
& a_{-} x=a \cos \beta \cos \alpha \\
& a_{-} y=a \cos \beta \sin \alpha \\
& a_{-} z=a \sin \beta
\end{aligned}
$$

The displacement of three axis $(x, y, z)$ is

$$
\begin{aligned}
& s_{-} x=\frac{1}{2} a_{-} x \cdot t^{2} \\
& s_{-} y=\frac{1}{2} a_{-} y \cdot t^{2} \\
& s_{-} z=\frac{1}{2} a_{-} z \cdot t^{2}
\end{aligned}
$$

$\mathrm{t}$ is the motion time of target. The displacement in the $O O_{1}$ direction is

$$
s_{2}=\operatorname{sqrt}\left(s_{-} x^{2}+s_{-} y^{2}+s_{-} z^{2}\right)
$$

So the position of the target in the space coordinate at any time is

$$
\begin{aligned}
& x=\left(s_{1}+s_{2}\right) \cos \beta \cos \alpha \\
& y=\left(s_{1}+s_{2}\right) \cos \beta \sin \alpha \\
& z=\left(s_{1}+s_{2}\right) \sin \beta
\end{aligned}
$$

\section{The Kalman Filtering Fusion of Location Informatio}

In this system, a Kalman-Filter is built by using an indirect method forestimating the position value. That is that the system converts the estimation of location information to the error of position information. After estimating the optimal error,error compensation would be done. As is shown in Figure 2.

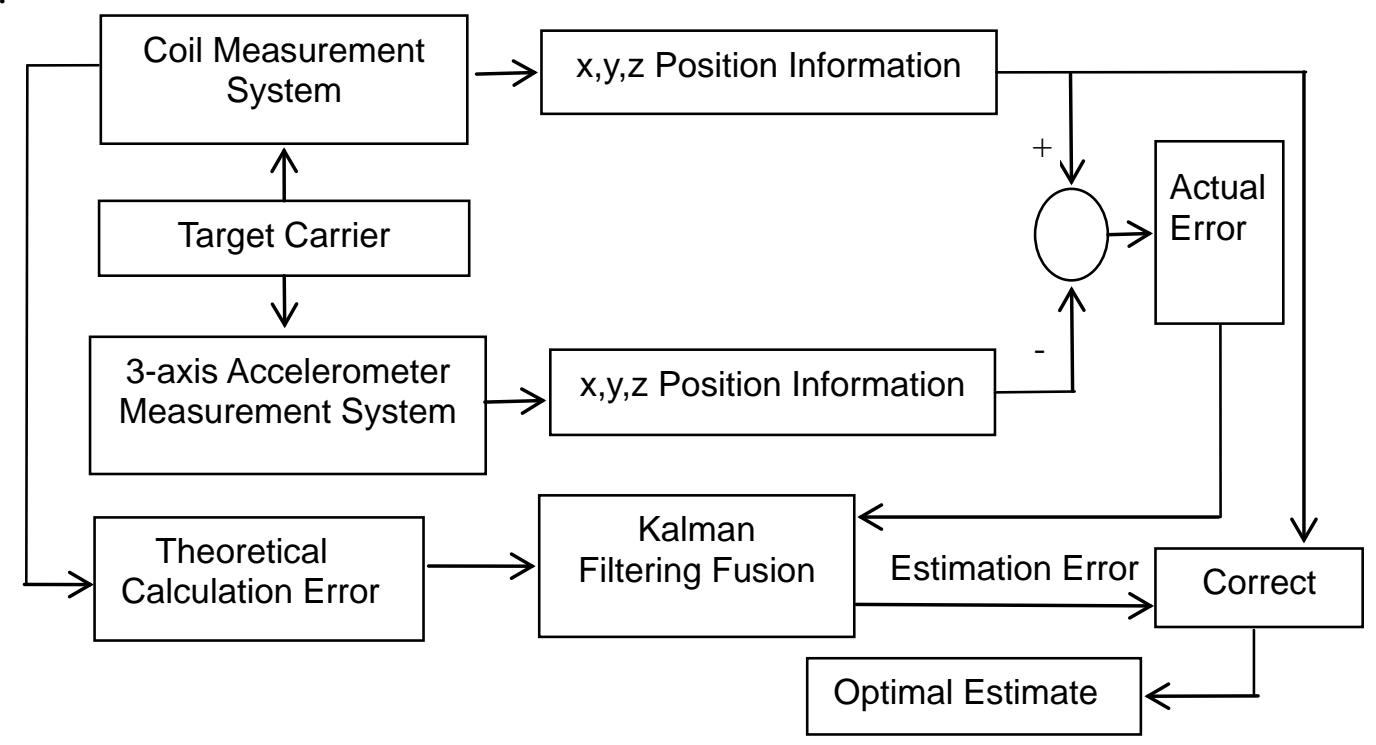

Fig.2. The detection of location information based on Kalman filtering fusion

The Kalman filtering theory allows us to obtain the real-time estimation based on the estimated value of previous time and measured value in real time. This is a recursive method and all the measurement data is used. In this paper, the difference of the position information between the 3-axis accelerometer system and the coil measurement system is used as the system measurement equation. The theoretical calculation error of the coil measurement system is used as the state equation of the system. After Kalman filtering fusion, the final estimation error would be obtained, which is used to correct the space position of target from the coil measurement system.

\section{Simulation Result}

During simulation, the emission source is placed at $O(0,0,0)$. The target moves in a straight line along a fixed direction. Then a set of space position data of target can be obtained in an ideal 
environment (without noise), which is set as the reference coordinate. There is noise interference in the real environment. So noise signal is added during simulation.Parameters setting in simulation: emission frequency of emission source is $20 \mathrm{khz}$,sampling frequency is $250 \mathrm{khz}$, the distance between the initial position of target and the emission source is $0.1 \mathrm{~m}$,the horizontal angle $\alpha=0.6 \mathrm{rad}$ and pitch angle $\beta=0.6$ rad.

As the distance between target and emission source increases, the bias of spatial coordinate measured by coil system is larger and larger. When the accelerometer measurement is introduced, and deal with the data from coil measurement system and 3-axis accelerometer measurement system with the filtering, the positioning accuracy has been greatly improved. The location information of simulation is shown in Figure 3.

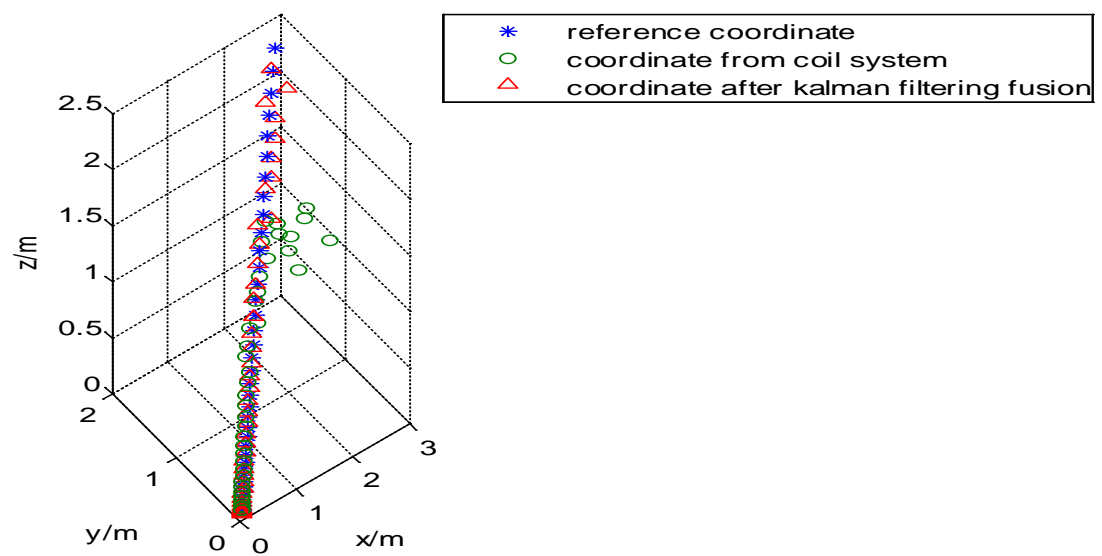

Fig.3. The space position of target

The simulation diagrams of error are shown in Figure 4 and Figure 5:
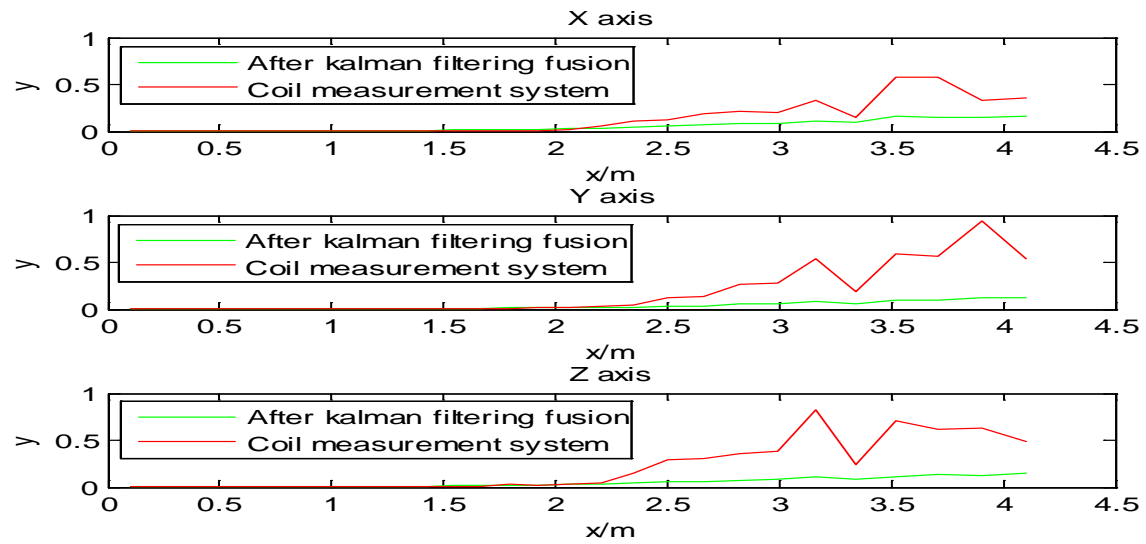

Fig.4. The comparison of mean-variance of error between coil measurement system and after Kalman filtering fusion 

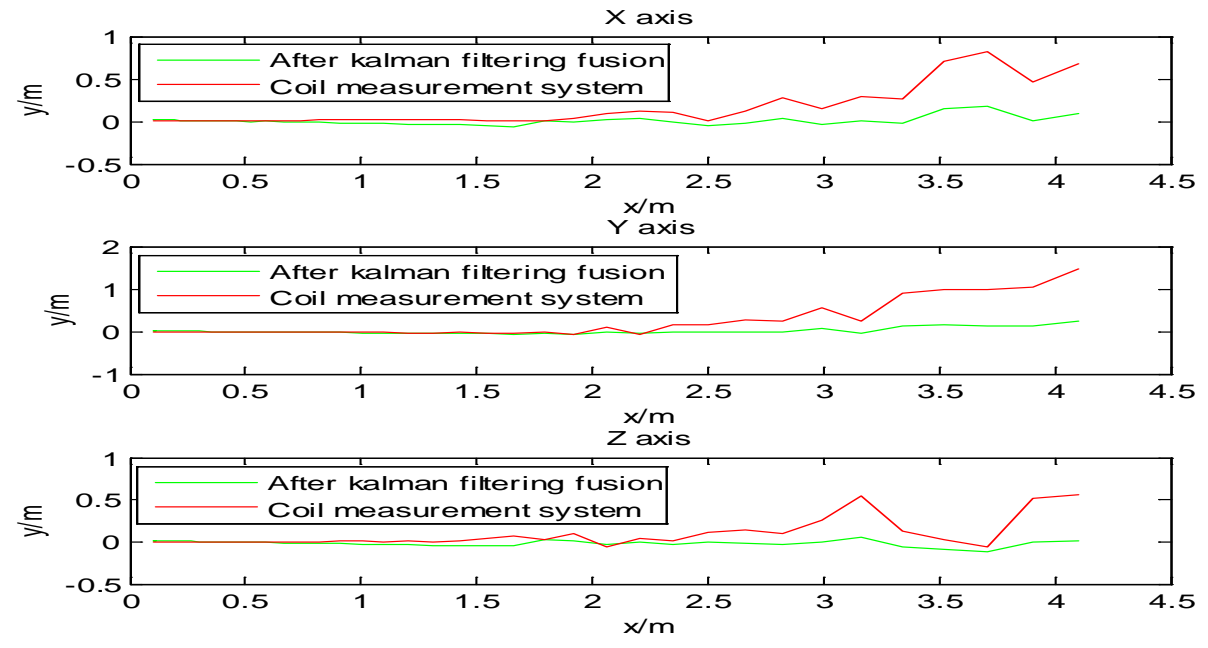

Fig.5. The comparison of mean value of error between coil measurement system and after Kalman filtering fusion

The Figure 4 and Figure 5 show the simulation diagrams of error. In Figure 4, the horizontal axis represents the distance between emission source and target, the vertical axis represents the mean-variance of positional error. And in Figure 5, the horizontal axis represents the distance between emission source and target, the vertical axis represents the mean value of positional error.

From Figure 4 and Figure 5, with the increase of distance, the mean-variance and mean value become larger obviously and fluctuate strongly of the coil system. The mean-variance and mean value of the position information being fused are small and are more stable. As shown in Figure 3, when $2.060 \mathrm{~m}<\rho<2.990 \mathrm{~m}$, the mean square error of the coil without Kalman fusion is 0.046, while the mean square error of the coil with Kalman fusion is 0.009 ; and when $2.990 \mathrm{~m}<\rho<4.100 \mathrm{~m}$, the mean square error of the coil without Kalman fusion is 0.149 , while the mean square error of the coil with Kalman fusion is 0.028 . It is obvious that the positioning precision of the electromagnetic positioning system have been greatly improved.

\section{Conclusion}

The Kalman fusion algorithm could fuse the electromagnetic positioning data and the accelerometer data, and enhance the positioning accuracy when the distance is lager. The simulation results show that when the distance is larger than $2.99 \mathrm{~m}$, the mean square error can be reduced to $18 \%$ of the original error.

\section{References}

[1] Tong Xu, Yongtian Wang, Dayuan Yan, Six Degree-of-Freedom Electromagnetic Tracking System for Virtual Reality, Journal of Beijing Institute of Technology, Vol.20,No.5,Walking pp.544-549, Oct.2000.

[2] Alfred M.Franz, Tamas Haidegger, Wolfgang Birkfellner, Electromagnetic Tracking In Medicine-A Review of Technology, Validation, and Applications, IEEE TRANSCATIONS ON MEDICAL IMAGING, VOL.33, NO.8, pp.1702-1725, Aug.2014.

[3] Qin Yin, Bin Chen ,Run Xiong , Zhaoyang Cai , Position Model of Six-DOF Electromagnetic Tracker and Near-Field Calibration Technique, Journal of Beijing University of Posts and Telecommunications, Vol.34,No.1,pp.64-68, Feb.2011.

[4] Ran Li, Cong Wang, Chaokun Feng ,A Method for Correcting Measurement Error of Electromagnetic Positioning System, Electronics Optics \& Control, Vol.21, No.4,pp.81-85, 
Apr.2014.

[5] Eugene Paperno, Ichiro Sasada, Eduard Leonovich, A New Method for Magnetic Position and Orientation Tracking[J],IEEE Transaction on Magnetics, July 2001,37(4):1938-1939.

[6] Yue Liu, Yongtian Wang, Xiaoming Hu, Study on Tracking Device with Pulsed AC Magnetic Field for Virtual Reality System[C], Proceeding of SPIE, 2003,4756:172-177. 